\title{
Microwave Tomography for Brain Stroke Imaging
}

\author{
P.-H. Tournier, F. Hecht, F. Nataf \\ UMPC, CNRS, LJLL; INRIA-Paris, EPC-Alpines \\ Paris, France \\ tournier@ann.jussieu.fr, hecht@ann.jussieu.fr, \\ nataf@ann.jussieu.fr
}

\author{
S. Semenov \\ EMTensor $\mathrm{GmbH}$ \\ Vienna, Austria \\ serguei.semenov@emtensor.com
}

\begin{abstract}
This paper deals with microwave tomography for brain stroke imaging using state-of-the-art numerical modeling and massively parallel computing. Iterative microwave tomographic imaging requires the solution of an inverse problem based on a minimization algorithm (e.g. gradient or Newton-like methods) with successive solutions of a direct problem. The solution direct requests an accurate modeling of the wholemicrowave measurement system as well as the as the whole-head. Moreover, as the system will be used for detecting brain strokes (ischemic or hemorrhagic) and for monitoring during the treatment, running times for the reconstructions should be fast. The method used is based on high-order finite elements, parallel preconditioners with the Domain Decomposition method and Domain Specific Language with open source FreeFEM++ solver.
\end{abstract}

Keywords-Microwave Tomography, Microwave Imaging, Brain Strokes, Inverse Problems, Parallel computing

\section{INTRODUCTION}

This paper deals with the detection and monitoring of brain stroke or cerebrovascular accident (CVA). About $85 \%$ of strokes are ischemic, caused by an interruption of the blood supply to some part of the brain and $15 \%$ are hemorrhagic. Differentiation between these two types of strokes is essential because the subsequent management and treatment of each patient is vastly different. Rapid and accurate diagnosis is crucial. CT and MRI are actually the "gold" standards but they are bulky diagnostic instruments and cannot be used for continuous brain monitoring. A non-invasive and transportable/portable device would have clear clinical applications at the bedside in a Neurological Intensive Care Unit (NICU).

Detecting and identifying strokes using microwave tomography is challenging as it corresponds to a small opposite variation of the permittivity values of brain tissues of about $+/$ $10 \%$ of the baseline tissue values for the two types of strokes (ischemic or hemorrhagic) [1]. The rapid data acquisition time is another attractive feature of microwave tomography but fast tomographic reconstructions are mandatory for developing a novel imaging modality with a new paradigm: detecting, identifying and monitoring stroke continuously during

This work was supported by the French ANR project MEDIMAX

\author{
M. Bonazzoli, F. Rapetti, V. Dolean \\ Université Côte d'Azur, CNRS, LJAD \\ Nice, France \\ marcella@bonazzoli.it, francesca.rapetti@unice.fr \\ victorita.dolean@unice.fr
}
I. El Kanfoud, I. Aliferis, C. Migliaccio, Ch. Pichot*
Université Côte d'Azur, CNRS, LEAT
Sophia Antipolis, France

ibtissam.el-kanfoud@unice.fr, iannis.aliferis@unice.fr claire.migliaccio@unice.fr, christian.pichot@unice.fr treatment. Iterative tomographic imaging requires the solution of an inverse problem based on a minimization algorithm. Reconstruction algorithms are computationally intensive with successive solutions of the forward problem needing efficient numerical modeling and high-performance parallel computing. The interaction is very complex, as it must be seen as a coupling problem between the antennas and the head rather than a simple scattering problem. In addition, we do not have access directly to the electric field but only via antenna $\mathrm{S}$ parameters. The purpose of this work is to solve the inverse problem associated to a prototype developed by EMTensor GmbH (Vienna, Austria) [2] using state-of-the-art modeling, high-performance and massively parallel computing.

\section{TOMOGRAPHIC SYSTEM}

The system consists of a cylindrical metallic chamber composed of 5 rings of 32 Transmitting/Receiving antennas (Fig. 1).
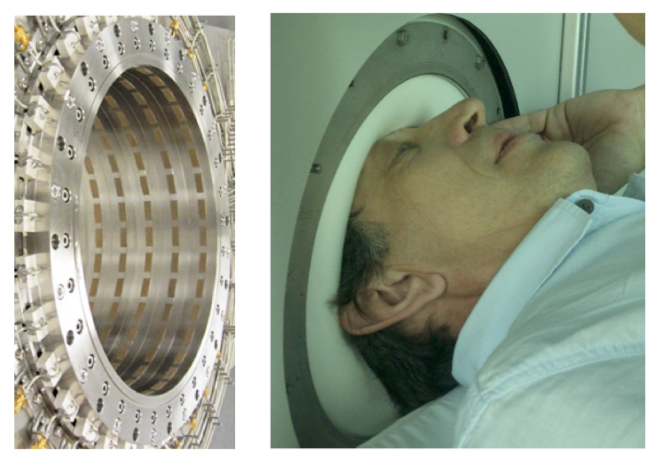

Fig. 1. Left: General view of BRIMG1 (courtesy of EMTensor

The antennas are ceramic $\left(\varepsilon_{r}=59\right)$ loaded open-ended waveguides. The operating frequency of the system is $0.9 \mathrm{GHz}$ to $1.8 \mathrm{GHz}$. The data acquisition cycle of the system is fully electronically controlled, allowing for a total data acquisition of about $30 \mathrm{~s}$. The imaging chamber is in horizontal position, 
allowing easy positioning a human head within an imaging domain (Fig. 1).

\section{DIRECT PROBLEM}

The numerical modeling of the direct problem is based on high-order finite elements, parallel predicontioners from Domain Decomposition method and Domain Specific Language with open source FreeFEM++ solver [3-4]. In order to have a higher numerical accuracy with the same total number of unknowns, we consider a high order edge element discretization, choosing the high order extension of Nédélec elements presented in [5]. We implemented edge elements of degrees 2 and 3 in FreeFem++. Domain decomposition preconditioners are naturally suited to parallel computing and make it possible to deal with smaller subproblems. The domain decomposition preconditioner we employ is called Optimized Restricted Additive Schwarz (ORAS).

\section{INVERSE PROBLEM}

The measured physical quantities are the $\mathrm{S}$ parameters of the scattering matrix, which are the complex reflection and transmission coefficients measured by the 160 receiving antennas when a signal is transmitted by one of the 160 transmitting antennas. The inverse problem that we consider consists in finding the unknown complex dielectric permittivity $\varepsilon(x)$ in order the corresponding scattering parameters $S$ from the forward modeling coincide with the measured scattering parameters $S_{i j}^{\text {meas }}$. In a classical way, solving the inverse problem consists in minimizing a cost functional $\mathrm{J}$. We use the adjoint approach in order to compute the gradient efficiently after discretization, with a number of computations independent of the size of the parameter space. We compute the gradient to use in a gradient-based local optimization algorithm. The numerical results presented here are obtained using a limited-memory Broyden-FletcherGoldfarb-Shanno (L-BFGS) algorithm.

\section{NUMERICAL RESULTS}

We want to assess the feasibility of the microwave imaging technique presented in this paper for stroke detection and monitoring through a numerical example in a realistic configuration. We use synthetic data corresponding to an accurate numerical model of a human head with a simulated hemorrhagic stroke as input for the inverse problem. The numerical model of the virtual head comes from CT and MRI scans and consists of a complex permittivity map of $362 \times 434$ $\times 362$ data points with a spatial resolution of $500 \mu$. In order to simulate the evolution of a hemorrhagic stroke, we use a synthetic ellipsoid-shaped stroke whose size (principal axes) increases over time, from $3.9 \mathrm{~cm} \times 2.3 \mathrm{~cm} \times 2.3 \mathrm{~cm}$ (small stroke) to $7.7 \mathrm{~cm} \times 4.6 \mathrm{~cm} \times 4.6 \mathrm{~cm}$ (large stroke). For this test case, the relative complex permittivity of the ellipsoid is assumed to be inhomogeneous where the relative complex permittivity at each quadrature point of the mesh is taken as the mean value between the original healthy brain permittivity values (baseline values) and the permittivity of blood ( $\varepsilon_{r}^{\text {blood }}=$ $68-\mathrm{i} 44)$ at $\mathrm{f}=1 \mathrm{GHz}$. The imaging chamber is filled with the matching solution $\varepsilon_{r}^{\text {matching }}=44-\mathrm{i} 20$. The synthetic data are obtained by solving the direct problem using a mesh composed of 17.6 million tetrahedra (corresponding to approximately 20 points $/ \lambda$ ) and consist in the computed transmission and reflection coefficients $S_{i j}$ with $10 \%$ noise.

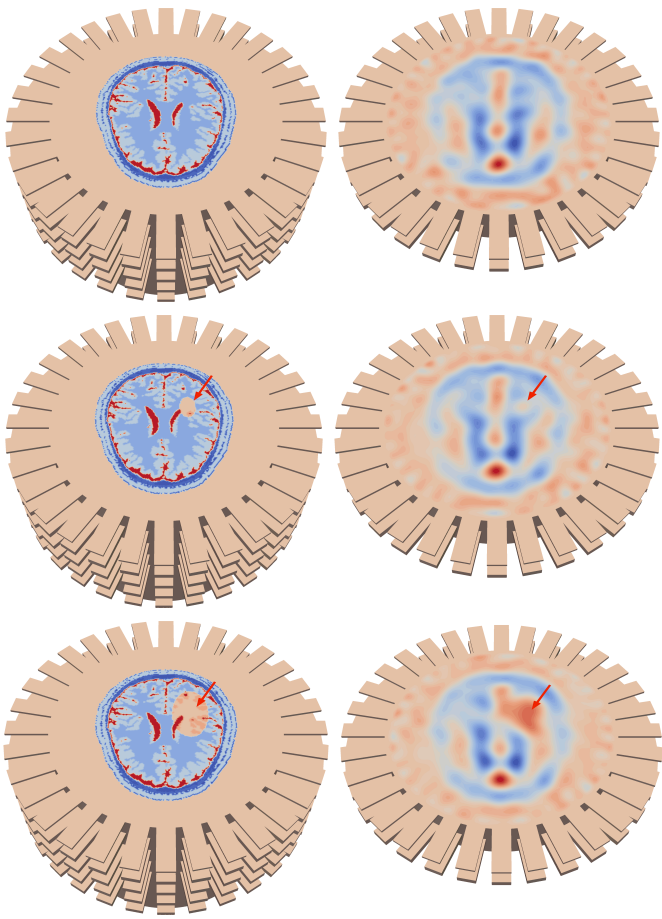

Fig. 2. Left: exact imaginary part of complex permittivity of a simulated hemorrhagic stroke (indicated with a red arrow) over time. Right: reconstructed imaginary part over time (after 30 iteration steps).

Reconstructed images for each test case shown in Fig. 2 are obtained with a total computing time of less than 2 minutes (94 seconds for the large stroke case) using 4096 cores of Curie.

\section{References.}

[1] S. Semenov, R. Svenson, V. Posukh, A. Nazarov, Y. Sizov, A. Bulyshev, A. Souvorov, W. Chen, J. Kassell, and G. Tatsis, "Dielectrical spectroscopy of canine myocardium during acute ischemia and hypoxia at frequency spectrum from $100 \mathrm{kHz}$ to $6 \mathrm{GHz}$," IEEE Trans. Med. Imag., Vol. 21, No.6, pp. 703-707, June 2002.

[2] S. Semenov, B. Seiser, E. Stoegmann, and E. Auff, "Electromagnetic tomography for brain imaging: from virtual to human brain," 2014 IEEE Conference on Antenna Measurements \& Applications (CAMA), Antibes Juan-les-Pins, 2014. Paper CAMA1162-SP132.4.pdf

[3] F. Hecht, "New development in FreeFem++," J. Numer. Math., vol. 20, no. 3-4, pp. 251-265, 2012.

[4] V. Dolean, P. Jolivet, and F. Nataf, An Introduction to Domain Decomposition Methods: algorithms, theory and parallel implementation. SIAM, 2015.

[5] F. Rapetti, "High order edge elements on simplicial meshes," $M 2 A N$ Math. Model. Numer. Anal., vol. 41, no. 6, pp. 1001-1020, 2007. 